\title{
Union-closed families with small average overlap densities
}

\author{
David Ellis \\ School of Mathematics \\ University of Bristol \\ Bristol, U.K. \\ david.ellis@bristol.ac.uk
}

Submitted: Dec 24, 2020; Accepted: Oct 17, 2021; Published: Jan 28, 2022

(C) The authors. Released under the CC BY-ND license (International 4.0).

\begin{abstract}
In this very short paper, we show that the average overlap density of a unionclosed family $\mathcal{F}$ of subsets of $\{1,2, \ldots, n\}$ may be as small as

$$
\Theta\left(\left(\log _{2} \log _{2}|\mathcal{F}|\right) /\left(\log _{2}|\mathcal{F}|\right)\right)
$$

for infinitely many positive integers $n$.

Mathematics Subject Classifications: 05D05

If $X$ is a set, a family $\mathcal{F}$ of subsets of $X$ is said to be union-closed if the union of any two sets in $\mathcal{F}$ is also in $\mathcal{F}$. The celebrated Union-Closed Conjecture (a conjecture of Frankl [2]) states that if $X$ is a finite set and $\mathcal{F}$ is a union-closed family of subsets of $X$ (with $\mathcal{F} \neq\{\varnothing\}$ ), then there exists an element $x \in X$ such that $x$ is contained in at least half of the sets in $\mathcal{F}$. Despite the efforts of many researchers over the last forty-five years, and a recent Polymath project [5] aimed at resolving it, this conjecture remains wide open. It has only been proved under very strong constraints on the ground-set $X$ or the family $\mathcal{F}$; for example, Balla, Bollobás and Eccles [1] proved it in the case where $|\mathcal{F}| \geqslant \frac{2}{3} 2^{|X|}$; more recently, Karpas [3] proved it in the case where $|\mathcal{F}| \geqslant\left(\frac{1}{2}-c\right) 2^{|X|}$ for a small absolute constant $c>0$; and it is also known to hold whenever $|X| \leqslant 12$ or $|\mathcal{F}| \leqslant 50$, from work of Vučković and Živković [8] and of Roberts and Simpson [7].

In 2016, a Polymath project [5] was convened to tackle the Union-Closed Conjecture. While it did not result in a proof of the conjecture, several interesting related conjectures were posed. Among them was the 'average overlap density conjecture'.
\end{abstract}

\section{Introduction}


If $X$ is a finite set and $\mathcal{F} \subset \mathcal{P}(X)$ with $\mathcal{F} \neq \varnothing$, we define the abundance of $x$ (with respect to $\mathcal{F}$ ) by $\gamma_{x}=|\{A \in \mathcal{F}: x \in A\}| /|\mathcal{F}|$, i.e., $\gamma_{x}$ is the probability that a uniformly random member of $\mathcal{F}$ contains $x$. A natural first quantity to consider, in trying to prove the Union-Closed Conjecture, is the average abundance of a uniformly random element of the ground set, i.e., $\mathbb{E}_{x \in X}\left[\gamma_{x}\right]$; if this quantity were always at least $1 / 2$, the UnionClosed Conjecture would immediately follow. A moment's thought shows that this is false, however, e.g. by considering the union-closed family $\{\varnothing,\{1\},\{1,2,3\}\} \subset \mathcal{P}(\{1,2,3\})$, which has average abundance $4 / 9$. Similarly, for any $n \in \mathbb{N}$, the union-closed family $\mathcal{F}=\{\varnothing,\{1\},\{1,2\}, \ldots,\{1,2, \ldots,\lfloor\sqrt{n}\rfloor\},\{1,2,3, \ldots, n\}\} \subset \mathcal{P}(\{1,2, \ldots, n\})$ has average abundance $\Theta(1 / \sqrt{n})=\Theta(1 /|\mathcal{F}|)$.

It is natural to consider the expected abundance of a random element of the groundset $X$ chosen according to other (non-uniform) distributions on $X$. The following was considered in the Polymath project [5]. We define the average overlap density $\operatorname{AOD}(\mathcal{F})$ of $\mathcal{F}$ to be the expected value of $\gamma_{x}$, where $x$ is a uniformly random element of a uniformly random nonempty member of $\mathcal{F}$ :

$$
\begin{aligned}
\operatorname{AOD}(\mathcal{F}) & :=\frac{1}{|\mathcal{F} \backslash\{\varnothing\}|} \sum_{A \in \mathcal{F} \backslash\{\varnothing\}} \frac{1}{|A|} \sum_{x \in A} \gamma_{x} \\
& =\frac{1}{|\mathcal{F} \backslash\{\varnothing\}|} \sum_{A \in \mathcal{F} \backslash\{\varnothing\}} \frac{1}{|A|} \sum_{x \in A} \frac{|\{B \in \mathcal{F}: x \in B\}|}{|\mathcal{F}|} \\
& =\frac{1}{|\mathcal{F} \backslash\{\varnothing\}|} \sum_{A \in \mathcal{F} \backslash\{\varnothing\}}\left(\frac{1}{|\mathcal{F}|} \sum_{B \in \mathcal{F}} \frac{|A \cap B|}{|A|}\right) \\
& =\mathbb{E}_{A \in \mathcal{F} \backslash\{\varnothing\}} \mathbb{E}_{B \in \mathcal{F}}\left[\frac{|A \cap B|}{|A|}\right] .
\end{aligned}
$$

(The first and second expectations in (1) are of course over a uniformly random element of $\mathcal{F} \backslash\{\varnothing\}$, and a uniformly random element of $\mathcal{F}$, respectively.) The last equality justifies the 'average overlap' terminology. The average overlap density conjecture stated that if $X$ is a finite set, and $\mathcal{F}$ is a union-closed family of subsets of $X$ with $\mathcal{F} \neq \varnothing$ and $\mathcal{F} \neq\{\varnothing\}$, then the average overlap density of $\mathcal{F}$ is at least $1 / 2$. Clearly, it would immediately imply the Union-Closed Conjecture.

Unfortunately, the average overlap density conjecture was quickly shown to be false (during the Polymath project [6]); an infinite sequence of union-closed families $\mathcal{F}_{n} \subset$ $\mathcal{P}(\{1,2, \ldots, n\})$ was constructed with $\operatorname{AOD}\left(\mathcal{F}_{n}\right)=7 / 15+o(1)$ as $n \rightarrow \infty$. However, the following weakening of the average overlap density conjecture remained open.

Conjecture 1. There exists an absolute positive constant $c>0$ such that the following holds. Let $n \in \mathbb{N}$ and let $\mathcal{F} \subset \mathcal{P}(\{1,2, \ldots n\})$ be union-closed with $\mathcal{F} \neq\{\varnothing\}$. Then the average overlap density of $\mathcal{F}$ is at least $c$.

Conjecture 1 would immediately imply the weakening of the Union-Closed Conjecture where $1 / 2$ is replaced by the absolute positive constant $c$. In this very short paper, we prove the following. 
Theorem 2. For infinitely many positive integers $n$, there exists a union-closed family $\mathcal{F}$ of subsets of $\{1,2, \ldots, n\}$ whose average overlap density is $\Theta\left(\left(\log _{2} \log _{2}|\mathcal{F}|\right) /\left(\log _{2}|\mathcal{F}|\right)\right)$.

This disproves Conjecture 1 in a strong sense. It follows from an old result of Knill [4] that if $\mathcal{F} \subset \mathcal{P}(\{1,2, \ldots, n\})$ is union-closed, then there exists $x \in\{1,2, \ldots, n\}$ with abundance $\gamma_{x}=\Omega\left(1 /\left(\log _{2}|\mathcal{F}|\right)\right)$, so the average overlap density can, in the best-case scenario, only be used to improve this lower bound by a factor of $\Theta\left(\log _{2} \log _{2}|\mathcal{F}|\right)$.

\section{Proof of Theorem 2}

For $n \in \mathbb{N}$, we write $[n]:=\{1,2, \ldots, n\}$ for the standard $n$-element set, and if $\mathcal{G} \subset \mathcal{P}(X)$, the union-closed family generated by $\mathcal{G}$ is defined to be the smallest union-closed family of subsets of $X$ that contains $\mathcal{G}$.

Let $k, m, s \in \mathbb{N}$ with $s \leqslant k-2$ and $m \geqslant 2$, and let $n=k m$. Partition $[n]$ into $m$ sets $B_{1}, \ldots, B_{m}$ with $\left|B_{i}\right|=k$ for all $i$; in what follows, we will refer to the $B_{i}$ as 'blocks'. For each $i \in[m]$, choose a subset $T_{i} \subset B_{i}$ with $\left|T_{i}\right|=s$, and let $T=\cup_{i=1}^{m} T_{i}$. Now let $\mathcal{F} \subset \mathcal{P}([n])$ be the union-closed family generated by $\left\{B_{i} \cup\{j\}: i \in[m], j \in T\right\}$. Note that every set in $\mathcal{F}$ contains at least one block. The number of sets in $\mathcal{F}$ containing exactly one block is $m 2^{(m-1) s}$, and in general, for each $j \in[m]$, number $N_{j}$ of sets in $\mathcal{F}$ containing exactly $j$ blocks is $\left(\begin{array}{c}m \\ j\end{array}\right) 2^{(m-j) s}$, so

$$
N:=|\mathcal{F}|=\sum_{j=1}^{m} N_{j}=2^{(m-1) s} \sum_{j=1}^{m}\left(\begin{array}{c}
m \\
j
\end{array}\right) 2^{-(j-1) s} .
$$

For each $j \in[m]$, define $p_{j}:=N_{j} / N$; this is of course the probability that a uniformly random member of $\mathcal{F}$ contains exactly $j$ blocks. We note that

$$
\frac{p_{j+1}}{p_{j}}=\frac{N_{j+1}}{N_{j}}=\frac{m-j}{j+1} 2^{-s} \leqslant m 2^{-s} \quad \forall j \in[m-1] .
$$

Write $\tau:=m 2^{-s}$. For any $x \in[n] \backslash T$, we clearly have

$$
\gamma_{x}=\frac{1}{m} \sum_{j=1}^{m} j p_{j}
$$

since the conditional probability that $x$ is contained in a random member $A$ of $\mathcal{F}$, given that $A$ contains exactly $j$ blocks, is $j / m$. We have $p_{j} \leqslant \tau^{j-1} p_{1}$ for all $j \in[m]$, and therefore for any $x \in[n] \backslash T$, we have

$$
\frac{1}{m} \leqslant \gamma_{x} \leqslant \frac{1}{m}\left(1+2 \tau+3 \tau^{2}+\ldots+m \tau^{m-1}\right) \leqslant \frac{1}{m}(1+4 \tau) \leqslant \frac{2}{m},
$$

provided $\tau=m 2^{-s} \leqslant 1 / 4$. Now, every member $A$ of $\mathcal{F}$ contains at least one block, so for any member $A$ of $\mathcal{F}$, the probability a uniformly random element of $A$ is in $T$, is at most 
$\frac{m s}{k}$. Crudely, we have $1 / 2 \leqslant \gamma_{x} \leqslant 1$ for all $x \in T$, since $A \mapsto A \cup\{x\}$ is an injection from $\{A \in \mathcal{F}: x \notin A\}$ to $\{A \in \mathcal{F}: x \in A\}$, for any $x \in T$. Hence, we have

$$
\frac{1}{m} \leqslant \operatorname{AOD}(\mathcal{F}) \leqslant\left(1-\frac{m s}{k}\right) \cdot \frac{2}{m}+\frac{m s}{k} \cdot 1 \leqslant \frac{2}{m}+\frac{m^{2} s}{n},
$$

again provided $\tau=m 2^{-s} \leqslant 1 / 4$. Now we wish to minimize the right-hand side of $(2)$, subject to the constraint $m 2^{-s} \leqslant 1 / 4$; clearly the optimal choice is to take $s=\left\lceil\log _{2} m\right\rceil+2$, which yields

$$
\frac{1}{m} \leqslant \operatorname{AOD}(\mathcal{F}) \leqslant \frac{2}{m}+\frac{m^{2} \log _{2} m}{n}+O\left(m^{2} / n\right) .
$$

It is clear that the optimal choice of $m$ to minimize the right-hand side of (3) is

$$
m=\Theta\left(\left(\frac{n}{\log _{2} n}\right)^{1 / 3}\right),
$$

yielding $\operatorname{AOD}(\mathcal{F})=\Theta\left(\left(\left(\log _{2} n\right) / n\right)^{1 / 3}\right)$. Since, with these choices, we have

$$
\log _{2}|\mathcal{F}|=\Theta\left(n^{1 / 3}\left(\log _{2} n\right)^{2 / 3}\right),
$$

it follows that

$$
\operatorname{AOD}(\mathcal{F})=\Theta\left(\frac{\log _{2} \log _{2}|\mathcal{F}|}{\log _{2}|\mathcal{F}|}\right)
$$

proving Theorem 2 .

We proceed to note two further properties of the above construction. Firstly, the average abundance of a uniformly random element of $[n]$ (with respect to $\mathcal{F}$ ) satisfies

$$
\mathbb{E}_{x \in[n]}\left[\gamma_{x}\right]=\Theta\left(\frac{\log _{2} \log _{2}|\mathcal{F}|}{\log _{2}|\mathcal{F}|}\right)
$$

Secondly, the family $\mathcal{F}$ constructed above does not separate the points of $[n]$. (We say a family $\mathcal{F} \subset \mathcal{P}([n])$ separates the points of $[n]$ if for any $i \neq j \in[n]$ there exists $A \in \mathcal{F}$ such that $|A \cap\{i, j\}|=1$. It is easy to see that, in attempting to prove the Union-Closed Conjecture, we may assume that the union-closed family in question separates the points of the ground set, and this assumption was adopted for much of the Polymath project [5].) However, it is easy to see that the union-closed family $\mathcal{F} \cup\{[n] \backslash\{j\}: j \in[n]\}$ has asymptotically the same average overlap density as $\mathcal{F}$ (and asymptotically the same average abundance as $\mathcal{F})$, and does separate the points of $[n]$.

\section{References}

[1] I. Balla, B. Bollobás and T. Eccles, Union-closed families of sets. J. Combin. Theory (Series A), 120 (2013), 531-544.

[2] D. Duffus, in: I. Rival (Ed.), Graphs and Order. Reidel, Dordrecht, Boston, 1985, p. 525 . 
[3] I. Karpas, Two Results on Union-Closed Families. Preprint, August 2017. arXiv: 1708.01434.

[4] E. Knill, Graph generated union-closed families of sets. Manuscript, September 1994. arXiv:9409215.

[5] Polymath11: Frankl's Union-Closed Conjecture. 1: Strengthenings, variants, potential counterexamples.

https://gowers . wordpress . com/2016/01/29/func1-strengtheningsvariants-potential-counterexamples/.

[6] Polymath11: Frankl's Union-Closed Conjecture. 2: More examples. https://gowers . wordpress . com/2016/02/08/func2-more-examples/.

[7] I. Roberts and J. Simpson, A note on the union-closed sets conjecture. Australas. J. Combin., 47 (2010), 265-267.

[8] B. Vučković and M. Živković, The 12-element case of Frankl's conjecture. IPSI Transactions on Advanced Research, January 2017, Paper 9. 